\title{
Clinical Trial Registry Identifier
}

National Cancer Institute

\section{Source}

National Cancer Institute. Clinical Trial Registry Identifier. NCI Thesaurus. Code C98714.

A sequence of letters, numbers, or other characters that uniquely identifies a clinical trial within a clinical trial registry. 